\title{
Simulation of physical processes in devices with non-magnetic, milk-dispersed secondary part
}

\author{
Maksim Minkin ${ }^{1, *}$,Denis Kuimov ${ }^{1}$, and Andrej Mozgovoj ${ }^{2}$ \\ ${ }^{1}$ DSTU, Department of automation of production processes, 344000 Rostov-on-Don, Russia \\ ${ }^{2}$ DSTU, Department of safety and environmental protection, 344000 Rostov-on-Don, Russia
}

\begin{abstract}
At present, most machine-building enterprises focus on the problem of saving energy resources. The proposed system of air regeneration in industrial premises will allow to keep the air in the room at a comfortable temperature without removing it to the atmosphere. The second problem solved by this system is the prevention of pollutants entering the atmosphere. At the same time, the efficiency of this system is not inferior to analogs, and many of them even surpass. Energy costs are reduced by at least $30 \%$ because there is no need to use the system of regeneration of working elements. However, the development of this system requires the solution of a number of problems. One of the main problems is the control of the motion of particles in the corona discharge region. A number of differently directed forces act on a particle of a finely dispersed substance, which actually form the equation of motion of this particle. However, when composing the equation of particle motion, it is necessary to take into account the space charge in the outer region of the corona. With a fairly complex geometry of the core, it is impossible to take into account the influence of a volumetric charge analytically, and, consequently, the use of computer modeling tools is required. Another factor that needs to be taken into account is the appearance of an aerodynamic force arising from such a poorly understood phenomenon as the "ion wind" that appears in the presence of a corona discharge. The solution of the described problem will allow to create a system of air regeneration in industrial premises, allowing recirculation of polluted air without diversion into the atmosphere, which will reduce the cost of energy.
\end{abstract}

\section{Formulation of the problem. Construction of a mathematical model}

In general, the energy-saving air regeneration system in industrial premises is built on the basis of a standard system of shop ventilation. At the design stage, or in an already existing system, it is proposed to build in several additional elements. After the modernization of the system, its main working element is the electromechanical device for suppressing dust flows (electrostatic shutter). The scheme of the energy-saving air regeneration system in production facilities (Figure 1).

The problem of developing devices for blocking dust emissions requires a lot of attention, since the development of many industries, combined with conservation and rational use of its resources, is often closely associated with the problem of suppressing dustcontaining flows. The urgency of its solution increases with the increase of the territorial concentration of industrial enterprises and the increase in their individual capacities.

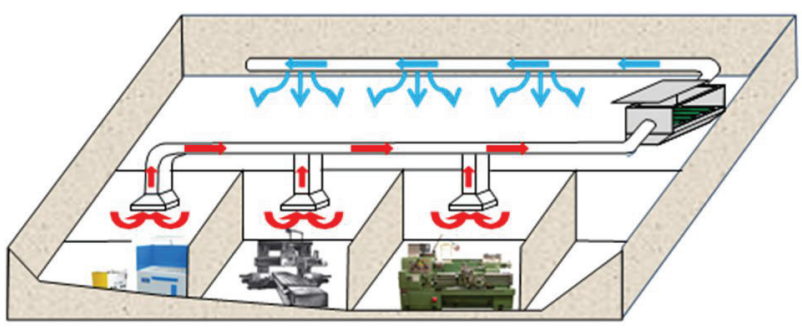

Fig. 1. Scheme of the energy-saving air regeneration system in production premises.

Under corona discharge conditions, the force exerted by the electric field acting on the particles of the finedispersed fraction is formed under the influence of the space charge, which affects the magnitude of this force $[1,2]$. Just take into account the aerodynamic effort, called the "ion wind," which in these devices, according to earlier experimental studies, is about $15-20 \%$ of the force from the side of the electric field. The distribution of finedispersed particles by physical properties and dimensions is also an important factor, since it affects the dynamics of particle acquisition and aerodynamic properties. The system of equations for the physical processes of particle motion in an inhomogeneous electric field, in general,

\footnotetext{
* Corresponding author: msi_58@mail.ru
} 
with an arbitrary moving part (aerosol cloud) is constructed on the basis of a mathematical model developed earlier by the leader of this project; however, in contrast to it, one can take into account the influence of the force acting on the part of the "ion wind ". The numerical solution of this system will allow predicting the behavior of a fine particle fraction, and based on the forecast, to develop an efficient system of air regeneration in industrial premises without air venting to the atmosphere.

To solve the problem, it is proposed to use computer simulation tools based on the finite element method. The computer model is based on a mathematical model describing physical processes in the core of an electromechanical device for blocking dust emissions. This mathematical model is constructed on the basis of the following regularities. The following forces act on a particle in an inhomogeneous electric field and under the influence of external perturbations: 1. Gravity; 2. The force of the electric bullet on a charged particle; 3 . The force due to the uneven distribution of the electric field strength; 4 . The strength of the resistance of the medium to the motion of particles; 5. Aerodynamic force generated by the "ion wind".

The system of equations for the physical processes of particle motion in an inhomogeneous electric field, in general, with an arbitrary moving part (aerosol cloud) is built on the basis of the mathematical model developed earlier by the project manager [1-5], but, unlike it, acting on the part of "ion wind" and includes the following equations:

$$
\begin{gathered}
\frac{d^{2} \mu}{d x^{2}}+\frac{d^{2} \mu}{d y^{2}}=-\frac{\rho}{\varepsilon_{0}} \\
\rho=\frac{d q(t)}{d v} \\
F_{1}=m g \\
F_{2}=q(t) E \\
F_{3}=\rho \frac{\pi r^{2}}{4} \\
F_{4}=\rho_{i \cdot w i n d} \frac{\pi r^{2}}{4} \\
m \frac{d V}{d t}=\sum F \\
q(t)=q_{m} \frac{e n_{0} k t}{4 \varepsilon_{0}+e n_{0} k t} \\
q_{m}=4 \pi \varepsilon_{0}\left(1+2 \frac{\varepsilon-1}{\varepsilon+2}\right) r^{2} E
\end{gathered}
$$

In this system of equations: $\varphi$ - is the scalar electric potential in the computational domain; $\rho$ - is the volumetric charge density; $q(\mathrm{t})$ - is the charge in the analyzed volume of the calculated region; $\mathrm{v}$ - is the volume of the investigated space; $\mathrm{m}$ - is the mass of the dust particle; qm - is the limiting charge of the dust particle; $p$ - air pressure in the dust-air mixture flow; p.vetra - air stream pressure created by the ion wind; $r$ radius of the dust particle.
Based on the analysis of the charging process of a particle as it moves in the core of the device, a preliminary evaluation of the operation of the electrostatic shutter can be made. In view of the fact that the physical properties of the finely dispersed fraction and the characteristics of the technological system have a great influence on the patterns of processes occurring in the interelectrode space of the dust-blocking devices, it is necessary to consider the operation of the devices based on the results given the characteristics of the finely dispersed fraction, and also the influence of the force acting with side of the "ion wind".

The numerical solution of this system will allow predicting the behavior of a particle of a finely dispersed fraction.

The process of the program for modeling physical processes begins with entering the initial data from the display screen:

1) the total number of nodes in the calculated area of a given section of the device;

2) the estimated number of nodes in the region of a given section;

3) geometric parameters of the electrostatic shutter, gate height, gate width;

As a result of the work of the above-described program, both the characteristics of the physical processes occurring in the electrostatic shutter and the characteristics of the processes affecting the behavior of the fine-particle fraction are obtained. A characteristic difference of this model of calculation of physical processes is the fact that, unlike all previously developed models, in the considered, it becomes possible to realize the modeling of the process of acquiring a charge by a particle of a finely dispersed fraction and a picture of the forces acting on the particle. This was possible, thanks to the introduction of special subprograms. In all previous studies, only the characteristics of the electric field were calculated, which did not give a complete picture of the processes taking place in electrostatic gates.

Based on the results of the simulation of electrical processes using the above computer model, it is determined:

1) characteristics of the electric field in each finite element of a given section of the device;

2) the dynamics of the acquisition of charge by a particle of a finely dispersed fraction;

3 ) the force acting on the particle of the finely dispersed fraction from the side of the electric field; In the framework of this work, a simulation of physical processes for several designs of dust-blocking devices was conducted, the results of the research were used in developing devices for specific operating conditions.

The study of physical processes is shown on the example of two variants of the electrostatic shutter design: "blindgrid", "comb-grid". The constructions of these devices are presented

The initial data, for all studies, were divided into the following groups.

1) Immutable technical data determined by the technological process:

- dust fraction - with diameter from 40 to 160 microns; 
- the temperature of the dust-air mixture, taking into account the reduction when entering an unheated zone $28^{\circ} \mathrm{C}$;

2) Immutable technical data determined by the parameters of the power supply network and the design features of the device under consideration:

- power is supplied from a single-phase network with a voltage $\mathrm{U}=220 \mathrm{~V} / 30 \mathrm{kV}$ with a frequency $\mathrm{f}=50 \mathrm{~Hz} / 0$ $\mathrm{Hz}$ (reverse polarity);

- cleaning of working parts - air.

In the considered versions of dust emission blocking devices, a number of physical processes have a direct effect on the efficiency of this type of device.

The quality of the device is the higher, the greater is the longitudinal component of the electric field at the entrance to the interelectrode space. The required configuration of the electric field lines at the entrance to the interelectrode space is achieved by a rational choice of the geometry of the shaping electrodes [6-9].

To the designer of the gate structure, it is necessary to achieve this direction and magnitude of the force vector acting on the side of the electric field in the active zone of the device, in which an efficient ejection of fine particles from the interelectrode space towards the total flow of the dust-air mixture occurs.

The blocking of dust emissions occurs in three stages:

1. charging of the fine-dispersed fraction of the dustair mixture;

2. inhibition of the dust fraction;

3. return of the dust fraction to the dust source or to a specially designated place for collecting the finely dispersed fraction.

To determine the design parameters of the device, under which its effective operation is ensured, it is necessary to use mathematical modeling of physical processes in the device.

It should be noted that in the study of physical processes in the "comb-grid" type of organization, modeling is performed proceeding from the assumption of a plane-parallel picture of the field in the interelectrode space [10-13]. Such an assumption becomes possible in the case when the distance between the electrodes belonging to the same comb is small enough to introduce significant distortions in the picture of the processes occurring.

For shutter-type "blind-mesh", in view of the design features, it makes sense to consider processes exclusively in the interelectrode window between the corona electrodes.

The simulation of physical processes in was performed with the change:

1) the interelectrode distance between the corona electrodes;

2) the diameter of a particle of a finely dispersed substance;

3) positions of the corona system with respect to the shaping electrode;

4) the values of the supply voltage from $30 \mathrm{kV}$ to $50 \mathrm{kV}$.

The obtained results of modeling the electric field made it possible to formulate the following recommendations for the construction of electrostatic gates.

1) the best results on the magnitude and nature of the variation of the longitudinal component of the electric field strength are obtained in the case when the points of the tip of the corona electrode and the points of intersection of the perpendiculars from the tip to the surface of the forming electrode lie at the vertices of an equilateral triangle;

2) on the particle of the fine-dispersed fraction, the effect from the side of the electric field balances the force of pressure from the side of the air flow, the faster, the more its geometric dimensions;

3) the presence of an additional shaping electrode at the entrance to the interelectrode space leads to an increase in the time of passage through the dust particles of the outer region of the corona, which increases the charge acquired by the particle;

4) the change in the interelectrode distance makes it possible to reduce the magnitude of the "dip" of the electric field strength along the symmetry axis between the corona electrodes;

5) the optimal distance between the corona and shaping electrodes should be selected from the calculation that the particle will travel from the entrance to the active zone of the electrostatic shutter to the guaranteed stop line in a time approximately equal to 0.1 seconds.

\section{Experimental research}

After obtaining theoretical results, necessary experimental studies were carried out to verify their adequacy. The abovementioned methodological approaches to conducting research to solve the tasks set in the project are implemented on the basis of a specially designed for this purpose experimental laboratory complex. To obtain a dust mixture, an experimental sample of the vortex layer activator is used, which allows processing of the material in a wide range. During the experimental research, the equipment of the Interdepartmental Resource Center for Collective Use (DMSTU) of the DSTU is included in the federal list of the Central Control Center of the Russian Federation, in particular, the centrifuge (CPS Disk Centrifuge DC24000) for sedimentation analysis of particulate matter of the aerosol mixture.The experimental data and the establishment of analytical dependencies using the methods of mathematical statistics, experiment planning, etc.

In the electrostatic gate, the shaping electrode is made in the form of a grid of non-magnetic material stretched perpendicular to the gas-air mixture flow and is connected to the positive pole of the high-voltage voltage source. The corona electrode is made of metal and consists of a series of angled plates at an equal distance from each other parallel to the shaping electrode and is connected to the negative pole of the power source. Such a design is very effective in view of the fact that an increase in the area of the corona electrodes, an increase in the residence time of the particle in the zone of the corona electrode, and also a partial mechanical removal of the dust fraction. 
In the devices under consideration, the fine particle moves in the interelectrode space due to excess air pressure. Then the force acting on the particle from the air side will be determined as the product of the excess air pressure inside the hopper or the container at the cross section of the particle. This force is counteracted by the complex of forces that act on it from the side of the electric field in the interelectrode space. Moreover, as the particle moves to the outlet, its charge increases and the braking force increases, while the force on the airflow side can be considered unchanged. The shutter will be considered to have performed its functions if the path traveled by the particle to a stop is less than the distance from the entry point of the particle to the outer corona discharge area to the hopper outlet [14-17].

The main physical processes ensuring the functioning of the shutter are: 1) the creation of a region of drift of electric charges in the space between the corona and forming electrodes; 2) adsorption of electric charges by finely dispersed particles; 3 ) deceleration of charged particles in an electric field; 4) redirection of the motion of charged dust particles. Thus, three stages can be distinguished in the working area of the device (Figure 2): 1) the region of particle charge; 2) area of inhibition; 3) the area of removal of particles from the working area.

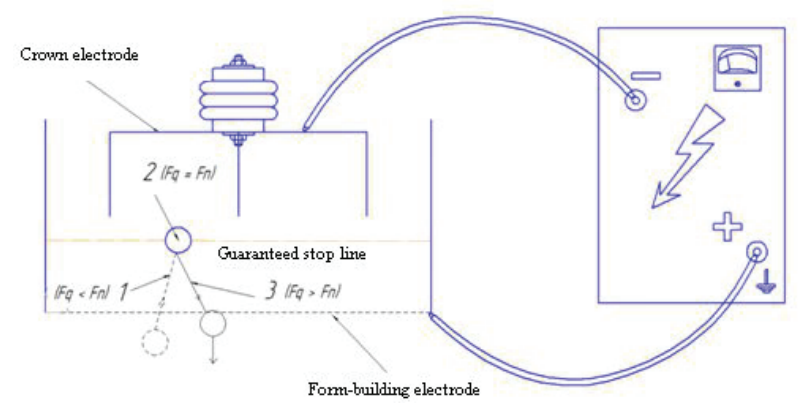

Fig. 2. Three stages of blocking dust emissions: 1) charging of the finely divided fraction of the dust-air mixture; 3 ) inhibition of the dust fraction; 2) return of the dust fraction to the source of dust or to a specially designated place for collecting the finely dispersed fraction.

To date, a large number of different devices for blocking dust emissions into the atmosphere have been developed, manufactured and used. They differ in a number of parameters, but most devices are air filtration systems, whereby filtration is carried out by depositing dust particles on the structural elements [18]. This method of cleaning affects the dimensions of the cleaning device and energy consumption for regeneration elements. The device, called an electrostatic shutter, performs a noncontact method of blocking a finely dispersed fraction in which the trapping and deposition of the dust fraction on the structural elements is not the main but a concomitant process [19-20].

The closest competitors of the electrostatic shutter are bag filters and electrostatic precipitators. The functional capabilities of the device proposed for examination are superior to known analogs (bag filters) or have similar characteristics (electrostatic precipitators) and provide a high efficiency of suppression of dust streams (degree of suppression of the dust fraction at least 95\%) with significantly reduced energy costs, which leads to the economization of valuable material and increase the efficiency of transportation of raw materials within the technological system. The selection of effective geometric parameters allows to reduce the size of devices for blocking dust emissions by 3-4 times and to reduce energy consumption by 5-10 times (up to 20 times). A high degree of efficiency of suppression of dust flows allows using these devices on an industrial scale (Fig. 3).

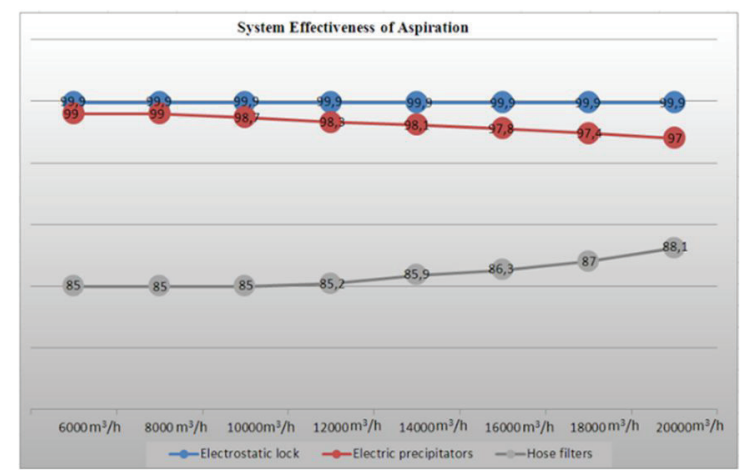

Fig. 3. The effectiveness of the aspiration system.

The essential advantage of an electrostatic shutter is its cost. On average, the cost of the electrostatic shutter is 1.5 times lower than the cost of a bag filter and 2 times lower than the cost of an electrostatic precipitator manufactured domestically. Thus, the total costs for the acquisition and operation of an electrostatic shutter are 15 to 20 times lower than the available analogs for the same cleaning efficiency (Figure 4).

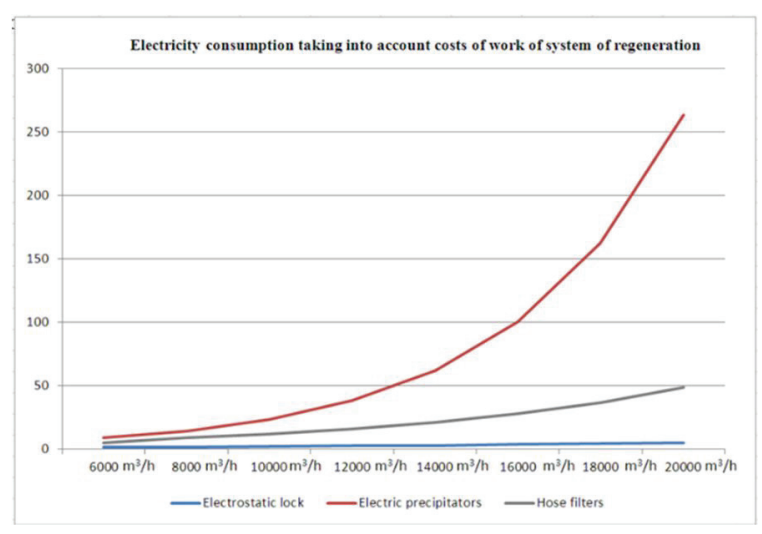

Fig. 4. Electricity consumption, taking into account the costs of the regeneration system.

On the basis of the conducted researches it is possible to assert that this device is effective and competitive in relation to analogues.

\section{Alternative applications of electromechanical dust suppression devices}

As an alternative application, one can consider the use of electromechanical devices as corona separation devices. Obviously, the underlying principles of these devices allow to broaden their scope of application provided that 
the method of controlling the behavior of the fine fraction in the active device area and use the ring separation electromechanical devices to select the fine fraction with the desired properties. At the present time, the most effective way of separating the finely dispersed fraction is electrical separation. A fairly large number of types of devices are known to divide the flow of a finely dispersed fraction.

Electrical separation is used to enrich granular bulk materials with a particle size of 3-0.05 $\mathrm{mm}$. Depending on the method of creating on the charge particles and its transfer in the process of electrical separation, there are: electrostatic, corona, dielectric, triboadhesive separation

Under corona discharge conditions, the force exerted by the electric field acting on the particles of the finedispersed fraction is formed under the influence of the space charge, which affects the magnitude of this force. We should also take into account the aerodynamic effort called "ion wind", which in these devices, according to earlier conducted experimental studies is about $15-20 \%$ of the force, from the side of the electric field. The distribution of fine-dispersed particles by physical properties and dimensions is also an important factor, since it affects the dynamics of particle acquisition and aerodynamic properties.

The system of equations for the physical processes of particle motion in an inhomogeneous electric field, in general, with an arbitrary moving part (aerosol cloud) is built on the basis of the mathematical model developed earlier by the project manager, but, unlike it, acting on the part of "ion wind" and includes the following equations:

$m \frac{d V}{d t}=\sum F$ - the equation of motion of the particle (1)

$F_{2}=q(t) E$ - Coulomb force acting on a charged particle (2)

$F_{1}=m g$-the particle force of a dust particle (3)

$F_{3}=\rho \frac{\pi r^{2}}{4} \quad$ - the force of excess air pressure (4)

$F_{4}=\frac{I_{k} \times h_{\text {м.э. }}}{k_{\text {ионов }}} \frac{\pi r^{2}}{4}$-the force created by the "ion" wind (5)

In this system of equations: $\varphi$ is the scalar electric potential in the computational domain, $\rho$ is the volumetric charge density, $\mathrm{q}(\mathrm{t})$ is the charge in the analyzed volume of the calculated region, $\mathrm{v}$ is the volume of the investigated space, $\mathrm{m}$ is the mass of the dust particle, $\mathrm{qm}$ is the limiting charge of the dust particle, $p$ - air pressure in the dust-air mixture flow, p.vetra - air stream pressure created by the ion wind, $\mathrm{r}$ - radius of the dust particle.

After substituting expressions (2-5) in (1), we obtain the following expression for the balance of forces:

$$
m \frac{d V}{d t}=m g+q(t) E+\left(\frac{I_{k} \times h_{\text {мэ. }}}{k_{\text {ионов }}}-\rho\right) \frac{\pi r^{2}}{4}
$$

The numerical solution of the given equation will allow to predict the behavior of a particle of a finely dispersed fraction. After receiving theoretical results, to verify their adequacy, it is necessary to conduct experimental studies.

Further studies of the physical characteristics of the electrostatic shutter are related to the study of the efficiency of work with a change in the particle size of the finely dispersed fraction passing through the active zone.
Investigation of the possibility of the shutter performing its functions was carried out at the moment the dust particle moved along the trajectory passing through the region of the least impact from the electric field. The corresponding trajectory of motion is found on the basis of the analysis of the characteristics of the magnitude of the force acting on a particle of matter along the guaranteed stop line. In this construction, this trajectory will coincide with the line of symmetry between the corona electrodes. The force acting on the dust fraction, as the core passes through the pre-selected trajectory, is shown in Fig. The investigations were carried out for particles with a diameter from $40 \mu \mathrm{m}$ to $160 \mu \mathrm{m}$. The calculated stopping point is indicated by the symbol $(\bullet)$.

Fqy, $\mathrm{H}$

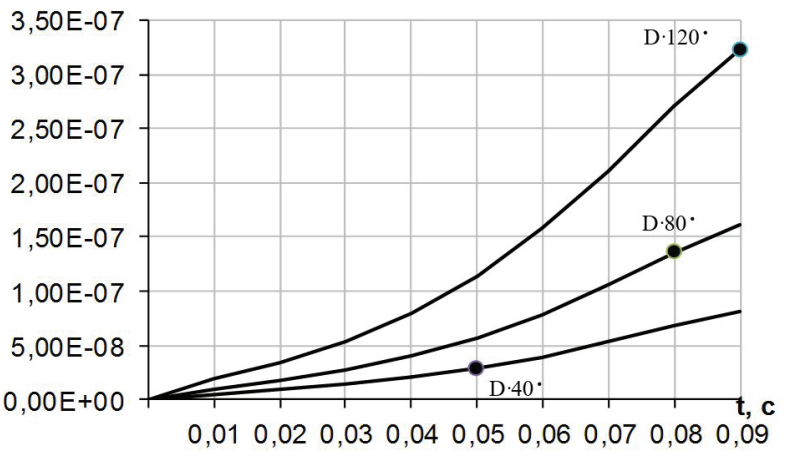

Fig. 5. Dynamics of the increase in force from the side of the electric field as the particle acquires a charge of a diameter from $40 \mu \mathrm{m}$ to $160 \mu \mathrm{m}$ as the core passes through the electrostatic shutter.

The study was carried out under the worst conditions for charging the particles. The analysis of the results obtained made it possible to conclude that the excess air pressure acting on the dust particle is balanced by the action from the electric field the faster the larger the geometric dimensions of the finely dispersed fraction. Modeling of physical processes in this design was carried out for a reverse polarity supply voltage of $30 \mathrm{kV}$ and a distance between the corona electrodes of $100 \mathrm{~mm}$.

\section{Conclusion}

The field of application of the electrostatic shutter is quite extensive, because the bulk of the pollutants are gaseous substances and finely dispersed materials that can travel for long distances and accumulate. The most demanded ESZ will be in the technological processes in the cement industry, metallurgy, brick and slate plants, for cleaning the air flow in the main electric locomotives and wagons, cleaning the smoke from solid fractions, cleaning the air in centralized air intakes of ventilation systems. For example, in the cement industry, the ESP can be used: for installation on the exhaust holes of clinker silos; when loading in road tankers; for installation on exhaust openings of cement silos; on the nodes of the transfer from the bucket conveyor; for post-treatment of the exhaust of the spike cooler; for loading wagons. 


\section{References}

1. M.S. Minkin, Electromechanical devices of blocking of dust emissions, Cand. tech. sciences. Diss., Novocherkassk, 144 (2013) [in Russian]

2. M.S. Minkin, G.I. Volodin, News of higher educational institutions. Electromecanics, no. 5, 33-38 (2013)

3. G.I. Volodin, V.I. Nadtoka, I.I. Nadtoka, V.I. Hludeev, P.F. Sidorenko, V.P. Breslavets, A.Y. Boasters, M.S. Minkin, Patent No.2438794, Russian Federation, MPK B03C3/00

4. G. I. Volodin, V. I. Nadtoka, I.I. Nadtoka, V.P. Breslavets, Nis Ya.Z., P.F. Sidorenko, M. S. Minkin, Patent No.107077, Russian Federation

5. M. S. Minkin, News of higher educational institutions. Electromecanics, no. 5, 61-66 (2010)

6. E. Karakas, A. Begum, M. Laroussi, A Positive, In Proc.IEEE Transactions on Plasma Science, 36(4), 950-951 (2008)

7. D. Lacoste, D. Pai, C. Laux, In Proc. 42nd AIAA Aerospace Sciences Meeting and Exhibit (2004)

8. R. Na, G. Fan, International Conference on Materials for Renewable Energy \& Environment 2014-2017 (2011)

9. R. Chengyan, W. Jue, Y. Ping, X. Rong, W. Tao, IEEE 11th International Conference on the Properties and Applications of Dielectric Materials,784-787 (2015)

10. V.L. Bychkov, V.A. Chernikov, S.A. Volkov, D.V. Bychkov, A.A. Kostiuk, J. IEEE Transactions on Plasma Science, no. 11, 2640-2641 (2011)

11. T. Johnson, S. Jakobsson, B. Wettervik, B. Andersson, A. Mark, F. Edelvik, Journal of Electrostatics, 27-36 (2015)

12. Y. Zhang, L. Liu, J. Ouyang, Journal of Electrostatics, no. $1,76-81$ (2014)

13. A. Iuga, R. Morar, A. Samuila, I. Cuglesan, M. Mihailescu, L. Dascalescu, J. IEEE Trans, on Ind. Appl, no.3 (1999)

14. R.Morar, A.Iuga, I.Cuglesan, O.Muntean, L.Dascalescu, J. IEEE Trans, on Ind. Appl, no.1 (1999)

15. G. Kazkaz, J. IEEE Trans, on Ind. Appl, no. 4 (1998)

16. A. Iuga, V. Neamtu, I. Suarasan, R. Morar, L. Dascalescu, J. IEEE Trans, on Ind. Appl, no. 2, 290326 (1998)

17. L. Dascalescu, D. Rafiroiu, A. Samuila, R. Tobazeon, J. IEEE Trans, on Ind. Appl, no. 1 (1998)

18. L. Daskalescu, R. Morar, A. Iuga, V. Neamtu, I. Suarasan, J. Magn. and Electrical Separ. No. 2, 91-106 (1993)
19. N. Szedenic, I. Kiss, 8 Int. Conf. on El.st., 88-92 (1997)

20. M.S. Minkin, A.D. Lukyanov, D.N. Kuimov S, Materials Science Forum, 625-629 (2016) 